\title{
On a Property of Non Liouville Numbers*
}

\author{
Jean-Marie De Koninck ${ }^{\dagger}$ and Imre Kátai ${ }^{\ddagger}$
}

Dedicated to the memory of Professor Ferenc Gécseg

\begin{abstract}
Let $\alpha$ be a non Liouville number and let $f(x)=\alpha x^{r}+a_{r-1} x^{r-1}+\cdots+$ $a_{1} x+a_{0} \in \mathbb{R}[x]$ be a polynomial of positive degree $r$. We consider the sequence $\left(y_{n}\right)_{n \geq 1}$ defined by $y_{n}=f(h(n))$, where $h$ belongs to a certain family of arithmetic functions and show that $\left(y_{n}\right)_{n \geq 1}$ is uniformly distributed modulo 1 .
\end{abstract}

Keywords: non Liouville numbers, uniform distribution modulo 1

\section{Introduction and notation}

Let $t(n)$ be an arithmetic function and let $f \in \mathbb{R}[x]$ be a polynomial. Under what conditions is the sequence $(f(t(n)))_{n \geq 1}$ uniformly distributed modulo 1 ? In the particular case where $f$ is of degree one, the problem is partly solved. For instance, it is known that, if $\alpha$ is an irrational number and if $t(n)=\omega(n)$ or $\Omega(n)$, where $\omega(n)$ stands for the number of distinct prime factors of $n$ and $\Omega(n)$ for the number of prime factors of $n$ counting their multiplicity, with $\omega(1)=\Omega(1)=0$, then the sequence $(\{\alpha t(n)\})_{n>1}$ is uniformly distributed modulo 1 (here $\{y\}$ stands for the fractional part of $y$ ). In 2005, we [1] proved that if $\alpha$ is a positive irrational number such that for each real number $\kappa>1$ there exists a positive constant $c=c(\kappa, \alpha)$ for which the inequality $\|\alpha q\|>c / q^{\kappa}$ holds for every positive integer $q$, then the sequence $(\{\alpha \sigma(n)\})_{n \geq 1}$ is uniformly distributed modulo 1. (Here $\|x\|$ stands for the distance between $x$ and the nearest integer and $\sigma(n)$ stands for the sum of the positive divisors of $n$.) Observe that one can construct an irrational number $\alpha$ for which the corresponding sequence $(\{\alpha \sigma(n)\})_{n \geq 1}$ is not uniformly distributed modulo 1 . On the other hand, given an integer $q \geq 2$ and letting $s_{q}(n)$ stand for the sum of the digits of $n$ expressed in base $q$, it is not hard to prove that, if $\alpha$ is an irrational number, the sequence $\left(\left\{\alpha s_{q}(n)\right\}\right)_{n \geq 1}$ is uniformly distributed modulo 1 . In fact, in the past 15 years, important results have been obtained concerning the

\footnotetext{
*The research of the first author was supported in part by a grant from NSERC.

†Département de mathématiques et de statistique Université Laval, Québec G1V 0A6, Canada. E-mail: jmdk@mat.ulaval.ca

¥Computer Algebra Department, Eötvös Loránd University, 1117 Budapest, Pázmány Péter Sétány I/C, Hungary. E-mail: katai@inf .elte.hu
} 
topic of the so-called $q$-ary arithmetic functions. For instance, it was proved that the sequence $\left(\left\{\alpha s_{q}(p)\right\}\right)_{p \in \wp}$ (here $\wp$ is the set of all primes) is uniformly distributed modulo 1 if and only if $\alpha \in \mathbb{R} \backslash \mathbb{Q}$. In 2010, answering a problem raised by Gelfond [10] in 1968, Mauduit and Rivat [13] proved that the sequence $\left(\left\{\alpha s_{q}\left(n^{2}\right)\right\}\right)_{n \geq 1}$ is uniformly distributed modulo 1 if and only if $\alpha \in \mathbb{R} \backslash \mathbb{Q}$.

Recall that an irrational number $\beta$ is said to be a Liouville number if for all integers $m \geq 1$, there exist two integers $t$ and $s>1$ such that

$$
0<\left|\beta-\frac{t}{s}\right|<\frac{1}{s^{m}}
$$

Hence, Liouville numbers are those real numbers which can be approximated "quite closely" by rational numbers.

Here, if $\alpha$ is a non Liouville number and

$$
f(x)=\alpha x^{r}+a_{r-1} x^{r-1}+\cdots+a_{1} x+a_{0} \in \mathbb{R}[x] \quad \text { is of degree } r \geq 1,
$$

we prove that $(f(t(n)))_{n \geq 1}$ is uniformly distributed modulo 1 , for those arithmetic functions $t(n)$ for which the corresponding function $a_{N, k}:=\frac{1}{N} \#\{n \leq N: t(n)=k\}$ is "close" to the normal distribution as $N$ becomes large.

Given $\mathcal{P} \subseteq \wp$, let $\Omega_{\mathcal{P}}(n)=\sum_{\substack{p^{r} \|_{n} \\ p \in \mathcal{P}}} r$. From here on, we let $q \geq 2$ stand for a fixed integer. Now, consider the sequence $\left(y_{n}\right)_{n \geq 1}$ defined by $y_{n}=f(h(n))$, where $h(n)$ is either one of the five functions

$$
\omega(n), \quad \Omega(n), \quad \Omega_{\mathcal{P}}(n), \quad s_{q}(n), \quad s_{q}\left(n^{2}\right) .
$$

Here, we show that the sequence $\left(y_{n}\right)_{n \geq 1}$ is uniformly distributed modulo 1 .

For the particular case $h(n)=s_{q}(n)$, we also examine an analogous problem, as $n$ runs only through the primes. Finally, we consider a problem involving strongly normal numbers.

Recall that the discrepancy of a set of $N$ real numbers $x_{1}, \ldots, x_{N}$ is the quantity

$$
D\left(x_{1}, \ldots, x_{N}\right):=\sup _{[a, b) \subseteq[0,1)}\left|\frac{1}{N} \sum_{\left\{x_{\nu}\right\} \in[a, b)} 1-(b-a)\right| .
$$

For each positive integer $N$, let

$$
M=M_{N}=\left\lfloor\delta_{N} \sqrt{N}\right\rfloor, \quad \text { where } \delta_{N} \rightarrow 0 \text { and } \delta_{N} \log N \rightarrow \infty \text { as } N \rightarrow \infty .
$$

We shall say that an infinite sequence of real numbers $\left(x_{n}\right)_{n \geq 1}$ is strongly uniformly distributed mod 1 if

$$
D\left(x_{N+1}, \ldots, x_{N+M}\right) \rightarrow 0 \quad \text { as } \quad N \rightarrow \infty
$$

for every choice of $M$ (and corresponding $\delta_{N}$ ) satisfying (3). Then, given a fixed integer $q \geq 2$, we say that an irrational number $\alpha$ is a strongly normal number 
in base $q$ (or a strongly $q$-normal number) if the sequence $\left(x_{n}\right)_{n \geq 1}$, defined by $x_{n}=\left\{\alpha q^{n}\right\}$, is strongly uniformly distributed modulo 1 . The concept of strong normality was recently introduced by De Koninck, Kátai and Phong [2].

We will at times be using the standard notation $e(x):=\exp \{2 \pi i x\}$. Finally, we let $\varphi$ stand for the Euler totient function.

\section{Background results}

The sum of digits function $s_{q}(n)$ in a given base $q \geq 2$ has been extensively studied over the past decades. Delange [4] was one of the first to study this function. Drmota and Rivat [7], [14] studied the function $s_{q}\left(n^{2}\right)$ and then, very recently, Drmota, Mauduit and Rivat [9] analyzed the distribution of the function $s_{q}(P(n))$, where $P \in \mathbb{Z}[x]$ is a polynomial of a certain type.

Here, we state as propositions some other results and recall two relevant results of Halász and Kátai.

First, given an integer $q \geq 2$, we set

$$
\mu_{q}=\frac{q-1}{2}, \quad \sigma_{q}^{2}=\frac{q^{2}-1}{12} .
$$

Proposition 1. Let $\delta>0$ be an arbitrary small number and let $\varepsilon>0$. Then, uniformly for $\left|k-\mu_{q} \log _{q} N\right|<\frac{1}{\delta} \sqrt{\log _{q} N}$,

$$
\begin{aligned}
& \#\left\{n \leq N: s_{q}(n)=k\right\}= \\
& \quad \frac{N}{\sqrt{2 \pi \sigma_{q}^{2} \log _{q} N}}\left(\exp \left\{-\frac{\left(k-\mu_{q} \log _{q} N\right)^{2}}{2 \sigma_{q}^{2} \log _{q} N}\right\}+O\left(\frac{1}{\log ^{\frac{1}{2}-\varepsilon} N}\right)\right) .
\end{aligned}
$$

Proof. This result is in fact a particular case of Proposition 3 below.

Proposition 2. Let $\varepsilon>0$. Uniformly for all integers $k \geq 0$ such that $(k, q-1)=1$,

$$
\begin{aligned}
& \#\left\{p \leq N: s_{q}(p)=k\right\}= \\
& \quad \frac{q-1}{\varphi(q-1)} \frac{\pi(N)}{\sqrt{2 \pi \sigma_{q}^{2} \log _{q} N}}\left(\exp \left\{-\frac{\left(k-\mu_{q} \log _{q} N\right)^{2}}{2 \sigma_{q}^{2} \log _{q} N}\right\}+O\left(\frac{1}{\log ^{\frac{1}{2}-\varepsilon} N}\right)\right) .
\end{aligned}
$$

Proof. This is Theorem 1.1 in the paper of Drmota, Mauduit and Rivat [8].

Let $G=\left(G_{j}\right)_{j \geq 0}$ be a strictly increasing sequence of integers, with $G_{0}=1$. Then, each non negative integer $n$ has a unique representation as $n=\sum_{j \geq 0} \epsilon_{j}(n) G_{j}$ with integers $\epsilon_{j}(n) \geq 0$ provided that $\sum_{j<k} \epsilon_{j}(n) G_{j}<G_{k}$ for all integers $k \geq 1$. Then, the sum of digits function $s_{G}(n)$ is given by

$$
s_{G}(n)=\sum_{j \geq 0} \epsilon_{j}(n) .
$$


Setting $a_{N, k}:=\#\left\{n \leq N: s_{G}(n)=k\right\}$, consider the related sequence $\left(X_{N}\right)_{N \geq 1}$ of random variables defined by

$$
P\left(X_{N}=k\right)=\frac{a_{N, k}}{N},
$$

so that the expected value of $X_{N}$ and its variance are given by

$$
E\left[X_{N}\right]=\frac{1}{N} \sum_{n \leq N} s_{G}(n) \quad \text { and } \quad V\left[X_{N}\right]=\frac{1}{N} \sum_{n \leq N}\left(s_{G}(n)-E\left[X_{N}\right]\right)^{2} .
$$

Let us choose the sequence $\left(G_{j}\right)_{j \geq 0}$ as the particular sequence

$$
G_{0}=1, \quad G_{j}=\sum_{i=1}^{j} a_{i} G_{j-1}+1 \quad(j>0),
$$

where the $a_{i}$ 's are simply the positive integers appearing in the Parry $\alpha$-expansion (here $\alpha>1$ is a real number) of 1 , that is

$$
1=\frac{a_{1}}{\alpha}+\frac{a_{2}}{\alpha^{2}}+\frac{a_{3}}{\alpha^{3}}+\cdots
$$

It can be shown (see Theorem 2.1 of Drmota and Gajdosik [5]) that, for such a sequence $\left(G_{j}\right)_{j \geq 0}$, setting

$$
G(z, u):=\sum_{j=1}^{\infty}\left(\sum_{\ell=0}^{a_{j}-1} z^{\ell}\right) z^{a_{1}+\cdots+a_{j-1}} u^{j}
$$

and letting $1 / \alpha(z)$ denote the analytic solution $u=1 / \alpha(z)$ of the equation $G(z, u)=$ 1 for $z$ in a sufficiently small (complex) neighbourhood of $z_{0}=1$ such that $\alpha(1)=\alpha$, then,

$$
E\left[X_{N}\right]=\mu \frac{\log N}{\log \alpha}+O(1)
$$

and

$$
V\left[X_{N}\right]=\sigma^{2} \frac{\log N}{\log \alpha}+O(1)
$$

where

$$
\mu=\frac{\alpha^{\prime}(1)}{\alpha} \quad \text { and } \quad \sigma^{2}=\frac{\alpha^{\prime \prime}(1)}{\alpha}+\mu-\mu^{2} .
$$

Proposition 3. Let $G=\left(G_{j}\right)_{j \geq 0}$ be as in (6). If $\sigma^{2} \neq 0$, then, given an arbitrary small $\varepsilon>0$, uniformly for all integers $k \geq 0$,

$$
\begin{aligned}
& \#\left\{n \leq N: s_{G}(n)=k\right\}= \\
& \quad \frac{N}{\sqrt{2 \pi V\left[X_{N}\right]}}\left(\exp \left\{-\frac{\left(k-E\left[X_{N}\right]\right)^{2}}{2 V\left[X_{N}\right]}\right\}+O\left(\frac{1}{\log ^{\frac{1}{2}-\varepsilon} N}\right)\right) .
\end{aligned}
$$


Proof. This is Theorem 2.2 in the paper of Drmota and Gajdosik [5].

Let $a$ be a positive integer. Let $q=-a+i$ (or $q=-a-i)$ and set $Q=a^{2}+1$ and $\mathcal{N}=\{0,1, \ldots, Q-1\}$. It is well known that every Gaussian integer $z$ can be written uniquely as

$$
z=\sum_{\ell \geq 0} \epsilon_{\ell}(z) q^{\ell} \quad \text { with each } \epsilon_{\ell} \in \mathcal{N} .
$$

Then, define the sum of digits function $s_{q}(z)$ of $z \in \mathbb{Z}[i]$ in base $q$ as

$$
s_{q}(z)=\sum_{\ell \geq 0} \epsilon_{\ell}(z)
$$

Proposition 4. Let $\mathcal{A}$ be the set of those positive integers a for which if $p \mid q=$ $-a \pm i$ and $|p| \neq 1$, then $|p|^{2} \geq 689$. Let $\mathcal{D}_{N}=\{z \in \mathbb{C}:|z| \leq \sqrt{N}\} \cap \mathbb{Z}[i]$ or $\mathcal{D}_{N}=\{z \in \mathbb{C}:|\Re(z)| \leq \sqrt{N},|\Im(z)| \leq \sqrt{N}\} \cap \mathbb{Z}[i]$. Then, uniformly for all integers $k \geq 0$, we have

$$
\begin{aligned}
& \frac{1}{\# \mathcal{D}_{N}} \#\left\{z \in \mathcal{D}_{N}: s_{q}\left(z^{2}\right)=k\right\}= \\
& \quad \frac{Q(k, q-1)}{\sqrt{2 \pi \sigma_{Q}^{2} \log _{Q}\left(N^{2}\right)}}\left(\exp \left\{-\frac{\Delta_{k}^{2}}{2}\right\}+O\left(\frac{(\log \log N)^{11}}{\sqrt{\log N}}\right)\right),
\end{aligned}
$$

where

$$
\Delta_{k}=\frac{k-\mu_{Q} \log _{Q}\left(N^{2}\right)}{\sqrt{\sigma_{Q}^{2} \log _{Q}\left(N^{2}\right)}}, \quad \mu_{Q}=\frac{Q-1}{2}, \quad \sigma_{Q}^{2}=\frac{Q^{2}-1}{12} .
$$

Proof. This result is a simplified version of Theorem 4 in Morgenbesser [15].

Let $a \in \mathbb{N}$ and $q=-a+i \in \mathbb{Z}[i]$. Set $\mathcal{N}=\left\{0,1, \ldots, a^{2}\right\}$. Then, every $z \in \mathbb{Z}[i]$ can be written uniquely as

$$
z=\sum_{j \geq 0} \epsilon_{j}(z) q^{j} \quad \text { with each } \epsilon_{j}(z) \in \mathcal{N} .
$$

Let $L$ be a non negative integer and consider a function $F: \mathcal{N}^{L+1} \rightarrow \mathbb{Z}$ satisfying $F(0,0, \ldots, 0)=0$ and set

$$
s_{F}(z)=\sum_{j=-L}^{\infty} F\left(\epsilon_{j}(z), \epsilon_{j+1}(z), \ldots, \epsilon_{j+L}(z)\right)
$$

The following is due to Drmota, Grabner and Liardet [6]. 
Proposition 5. Under certain conditions on F stated in Corollary 3 in Drmota, Grabner and Liardet [6],

$$
\begin{aligned}
& \#\left\{z \in \mathbb{Z}[i]:|z|^{2}<N, s_{F}(z)=k\right\}= \\
& \frac{\pi N}{\sqrt{2 \pi \sigma^{2} \log _{|q|^{2}}} N} \exp \left\{-\frac{\left(k-\mu \log _{|q|^{2}} N\right)^{2}}{2 \sigma^{2} \log _{|q|^{2}} N}\right\}\left(1+O\left(\frac{1}{\sqrt{\log N}}\right)\right)
\end{aligned}
$$

uniformly for $\left|k-\mu \log _{|q|^{2}} N\right| \leq c \sqrt{\log _{|q|^{2}} N}$, where c can be taken arbitrarily large.

For any particular set of primes $\mathcal{P}$, let $E(x)=E_{\mathcal{P}}(x):=\sum_{\substack{p \leq x \\ p \in \mathcal{P}}} \frac{1}{p}$.

The following two results, which we state as propositions, are due respectively to Halász [11] and Kátai [12].

Proposition 6. (HALÁSZ) Let $0<\delta \leq 1$ and let $\mathcal{P}$ be a set of primes with corresponding functions $\Omega_{\mathcal{P}}(n)$ and $E(x)=E_{\mathcal{P}}(x)$. Then, assuming that $E(x) \rightarrow$ $\infty$ as $x \rightarrow \infty$, the estimate

$$
\sum_{\substack{n \leq x \\ \Omega_{\mathcal{P}}(n)=k}} 1=\frac{x E(x)^{k}}{k !} e^{-E(x)}\left\{1+O\left(\frac{|k-E(x)|}{E(x)}\right)+O\left(\frac{1}{\sqrt{E(x)}}\right)\right\}
$$

holds uniformly for all positive integers $k$ and real numbers $x \geq 3$ satisfying

$$
E(x) \geq \frac{8}{\delta^{3}} \quad \text { and } \quad \delta \leq \frac{k}{E(x)} \leq 2-\delta .
$$

Proposition 7. (KÁtAI) For $1 \leq h \leq x$, let

$$
\begin{array}{cc}
A_{k}(x, h):=\sum_{\substack{x \leq n \leq x+h \\
\omega(n)=k}} 1, \quad B_{k}(x):=\sum_{\substack{n \leq x \\
\omega(n)=k}} 1, \\
\delta_{k}(x, h):=\frac{A_{k}(x, h)}{h}-\frac{B_{k}(x)}{x}, \quad E(x, h):=\sum_{k=1}^{\infty} \delta_{k}^{2}(x, h) .
\end{array}
$$

Letting $\varepsilon>0$ be an arbitrarily small number and $x^{7 / 12+\varepsilon} \leq h \leq x$, then

$$
E(x, h) \ll \frac{1}{\log ^{2} x \cdot \sqrt{\log \log x}} .
$$

\section{Main results}

Theorem 1. Let $f(x)$ be as in (1), $h(n)$ be one of the five functions listed in (2) and $y_{n}:=f(h(n))$. Then, the sequence $\left(y_{n}\right)_{n \geq 1}$ is uniformly distributed modulo 1 . 
Theorem 2. Let $f(x)$ be as in (1). Then, the sequence $\left(z_{p}\right)_{p \in \wp}$, where $z_{p}:=$ $f\left(s_{q}(p)\right)$, is uniformly distributed modulo 1 .

Theorem 3. Let $Q \geq 2$ and $q \geq 2$ be fixed integers. Let $\alpha$ be a strongly $Q$ normal number. Let $g$ be a real valued continuous function defined on $[0,1]$ such that $\int_{0}^{1} g(x) d x=0$. Then,

$$
\lim _{N \rightarrow \infty} \frac{1}{N} \sum_{n=1}^{N} g\left(\alpha Q^{h(n)}\right)=0,
$$

where $h(n)=s_{q}(n)$ or $s_{q}\left(n^{2}\right)$. Moreover, letting $\pi(N)$ stand for the number of prime numbers not exceeding $N$, we have

$$
\lim _{N \rightarrow \infty} \frac{1}{\pi(N)} \sum_{p \leq N} g\left(\alpha Q^{s_{q}(p)}\right)=0 .
$$

The following corollary follows from estimate (7) of Theorem 3.

Corollary 1. With $\alpha$ and $h(n)$ as in Theorem 3, the sequence $\left(\alpha Q^{h(p)}\right)_{p \in \wp}$ is uniformly distributed modulo 1.

In light of Proposition 3, we have the following two corollaries.

Corollary 2. Let $G$ be as in (4). Then, letting $f$ be as in (1), the sequence $\left(\left\{f\left(s_{G}(n)\right)\right\}\right)_{n \geq 0}$ is uniformly distributed modulo 1.

Corollary 3. Let $G$ be as in (4). Then, if $\alpha$ is a strongly normal number in base $Q$, the sequence $\left(\left\{\alpha \cdot Q^{s_{G}(n)}\right\}\right)_{n \geq 0}$ is uniformly distributed modulo 1.

As a direct consequence of the Main Lemma and of Proposition 4, we have the following result.

Theorem 4. Let $\mathcal{D}_{N}$ be as in Proposition 4. Let $f$ be as in (1). For each $z \in \mathcal{D}_{N}$, set $y_{z}:=f\left(s_{q}\left(z^{2}\right)\right)$. Then, the discrepancy of the sequence $y_{z}$ tends to 0 as $N \rightarrow \infty$, that is

$$
D\left(y_{z}: z \in \mathcal{D}_{N}\right) \rightarrow 0 \quad \text { as } N \rightarrow \infty .
$$

Theorem 5. Let $\mathcal{D}_{N}$ be as in Proposition 4. Let $\alpha$ be a strongly normal number in base $Q$ and consider the sequence $\left(y_{z}\right)_{z \in \mathcal{D}_{N}}$. Then

$$
D\left(y_{z}: z \in \mathcal{D}_{N}\right) \rightarrow 0 \quad \text { as } N \rightarrow \infty \text {. }
$$

In line with Proposition 7, we have the following.

Theorem 6. Let $\varepsilon>0$ be a fixed number. Let $H=\left\lfloor x^{7 / 12+\varepsilon}\right\rfloor$ and set

$$
\pi_{k}([x, x+H]):=\#\{n \in[x, x+H]: \omega(n)=k\} .
$$

Let $f$ be as in (1) and set

$$
S(x)=\sum_{x \leq n \leq x+H} e(f(\omega(n))) .
$$

Then

$$
\frac{S(x)}{H} \rightarrow 0 \quad \text { as } x \rightarrow \infty .
$$




\section{Preliminary lemmas}

Lemma 1. Let $\alpha$ be a non Liouville number and let $f(x)$ be as in (1). Then,

$$
\sup _{U \geq 1} \frac{1}{N}\left|\sum_{n=U+1}^{U+N} e(f(n))\right| \rightarrow 0 \quad \text { as } N \rightarrow \infty .
$$

Proof. Since $\alpha$ is a non Liouville number, there exists a positive integer $\ell$ such that if $\tau$ is a fixed positive number and

$$
\left|\alpha-\frac{t}{s}\right| \leq \frac{1}{s \tau}, \quad(t, s)=1, \quad s \leq \tau
$$

then $\tau^{1 / \ell}<s$.

Vaughan ([16], Lemma 2.4) proved that if $\left|\alpha-\frac{t}{s}\right|<\frac{1}{s^{2}}$ and $K=2^{t-1}$, then, given any small number $\varepsilon>0$,

$$
\sum_{n=U+1}^{U+N} e(f(n)) \ll_{\varepsilon} N^{1+\varepsilon}\left(\frac{1}{s}+\frac{1}{N}+\frac{s}{N^{t}}\right)^{1 / K} .
$$

Now, choose $\tau=N^{t / 2}$ so that $N^{t / 2^{\ell}}<s<\tau$. It then follows from (9) that

$$
\sum_{n=U+1}^{U+N} e(f(n)) \ll N^{1-\delta},
$$

for some $\delta>0$ which depends only on $\varepsilon$ and $\ell$, thus completing the proof of Lemma 1 .

Using this result, we can establish our Main Lemma.

Lemma 2. (Main Lemma) For each positive integer $N$, let $\left(E_{N}(k)\right)_{k \geq 1}$ be a sequence of non negative integers called weights which, given any $\delta>0$, satisfies the following three conditions:

(a) $\sum_{k=1}^{\infty} E_{N}(k)=1$;

(b) there exists a sequence $\left(L_{N}\right)_{N \geq 1}$ which tends to infinity as $N \rightarrow \infty$ such that

$$
\limsup _{N \rightarrow \infty} \sum_{\substack{k=1 \\ \frac{\left|k-L_{N}\right|}{\sqrt{L_{N}}}>\frac{1}{\delta}}}^{\infty} E_{N}(k) \rightarrow 0 \quad \text { as } \delta \rightarrow 0
$$

(c) $\lim _{N \rightarrow \infty} \max _{\frac{\left|k-L_{N}\right|}{\sqrt{L_{N}}} \leq \frac{1}{\delta}} \max _{1 \leq \ell \leq \delta^{3 / 2}}\left|\frac{E_{N}(k+\ell)}{E_{N}(k)}-1\right|=0$. 
Moreover, let $\alpha$ and $f$ be as in (1) and let

$$
T_{N}(f):=\sum_{k=1}^{\infty} e(f(k)) E_{N}(k)
$$

Then,

$$
T_{N}(f) \rightarrow 0 \quad \text { as } N \rightarrow \infty .
$$

Proof. Let $\delta>0$ be fixed and set

$$
\begin{gathered}
S:=\left\lfloor\delta^{3 / 2} \sqrt{L_{N}}\right\rfloor, \quad t_{m}=\left\lfloor L_{N}\right\rfloor+m S \quad(m=1,2, \ldots), \\
U_{m}=\left[t_{m}, t_{m+1}-1\right] \quad(m=1,2, \ldots) .
\end{gathered}
$$

Let us now write

$$
T_{N}(f)=S_{1}(N)+S_{2}(N)
$$

where

$$
\begin{aligned}
S_{2}(N) & =\sum_{\left|k-L_{N}\right|>\frac{1}{\delta} \sqrt{L_{N}}} E_{k}(N) e(f(k)), \\
S_{1}(N) & =\sum_{|m| \leq 1 / \delta^{5 / 2}} \sum_{k \in U_{m}} E_{k}(N) e(f(k))=\sum_{|m| \leq 1 / \delta^{5 / 2}} S_{1}^{(m)}(N),
\end{aligned}
$$

say.

First observe that, by condition (b) above,

$$
\left|S_{2}(N)\right| \leq \sum_{\frac{\left|k-L_{N}\right|}{\sqrt{L} N}>\frac{1}{\delta}} E_{N}(k)=o(1) \quad \text { as } N \rightarrow \infty .
$$

On the other hand, it follows from condition (c) above and Lemma 1 that, as $N \rightarrow \infty$,

$$
\begin{aligned}
\left|S_{1}^{(m)}(N)\right| & \leq E_{t_{m}}(N)\left|\sum_{k \in U_{m}} e(f(k))\right|+o(1) \sum_{k \in U_{m}} E_{k}(N) \\
& =o(1) S E_{t_{m}}(N)+o(1) \sum_{k \in U_{m}} E_{k}(N)
\end{aligned}
$$

while

$$
\left|S E_{t_{m}}(N)-\sum_{k \in U_{m}} E_{k}(N)\right|=o(1) \sum_{k \in U_{m}} E_{k}(N) .
$$

Gathering these two estimates, we obtain that

$$
S_{1}(N) \rightarrow 0 \quad \text { as } N \rightarrow \infty
$$

Using (12) and (13) in (11), conclusion (10) follows. 
Lemma 3. For each integer $k \geq 1$, let

$$
\begin{aligned}
& \pi_{k}(x):=\quad \#\{n \leq x: \omega(n)=k\}, \\
& \pi_{k}^{*}(x):=\quad \#\{n \leq x: \Omega(n)=k\}
\end{aligned}
$$

Then, the relations

$$
\begin{aligned}
& \pi_{k}(x)=(1+o(1)) \frac{x}{\log x} \frac{(\log \log x)^{k-1}}{(k-1) !} \\
& \pi_{k}^{*}(x)=(1+o(1)) \frac{x}{\log x} \frac{(\log \log x)^{k-1}}{(k-1) !}
\end{aligned}
$$

hold uniformly for

$$
|k-\log \log x| \leq \frac{1}{\delta_{x}} \sqrt{\log \log x},
$$

where $\delta_{x}$ is some function of $x$ chosen appropriately and which tends to 0 as $x \rightarrow \infty$.

Proof. This follows from Theorem 10.4 stated in the book of De Koninck and Luca $[3]$.

\section{Proof of Theorem 1}

We first consider the case when $h(n)$ is one of the three functions $\omega(n), \Omega(n)$ and $\Omega_{E}(n)$. Set

$$
\begin{aligned}
& \pi_{k}(N)=\#\{n \leq N: \omega(n)=k\}, \\
& \pi_{k}^{*}(N)=\#\{n \leq N: \Omega(n)=k\}, \\
& T_{k}(N)=\#\left\{n \leq N: \Omega_{E}(n)=k\right\} .
\end{aligned}
$$

In light of Lemma 3 and Proposition 6 , the corresponding weights of the sequences $\left(\pi_{k}(N)\right)_{k \geq 1},\left(\pi_{k}^{*}(N)\right)_{k \geq 1}$ and $\left(T_{k}(N)\right)_{k \geq 1}$ are $\pi_{k}(N) / N, \pi_{k}^{*}(N) / N$ and $T_{k}(N) / N$, respectively.

Now, in order to obtain the conclusion of the Theorem, we only need to prove that, for each non zero integer $m$,

$$
\frac{1}{N} \sum_{n \leq N} e(m f(h(n))) \rightarrow 0 \quad \text { as } N \rightarrow \infty .
$$

But this is guaranteed by Lemma 1 if we take into account the fact that since $\alpha$ is a non Liouville number, the number $m \alpha$ is also non Liouville for each $m \in \mathbb{Z} \backslash\{0\}$. Hence, the theorem is proved. 


\section{Proof of Theorem 2}

We cannot make a direct use of Lemma 2 because the estimate in that lemma only holds for those positive integers $k$ such that $(k, q-1)=1$. To avoid this obstacle, we shall subdivide the positive integers $k$ according to their residue class modulo $q-1$. Observe that there are $\varphi(q-1)$ such classes. Hence, we write each $k$ as

$$
k=t(q-1)+\ell, \quad(\ell, q-1)=1 .
$$

Hence, for each positive integer $\ell$ such that $(\ell, q-1)=1$, we set

$$
\wp_{\ell}:=\left\{p \in \wp: s_{q}(p) \equiv \ell \quad(\bmod q-1)\right\}, \quad \Pi_{\ell}(N):=\#\left\{p \leq N: p \in \wp_{\ell}\right\} .
$$

It is easy to verify that

$$
\frac{\Pi_{\ell}(N)}{\pi(N)}=(1+o(1)) \frac{1}{\varphi(q-1)} \quad(N \rightarrow \infty) .
$$

Thus, in order to prove Theorem 2, we need to show that the sum

$$
U_{\ell}(N):=\sum_{\substack{p \leq N \\ s_{q}(p) \equiv \ell(\bmod q-1)}} e\left(m f\left(s_{q}(p)\right)\right),
$$

where $m$ is any fixed non zero integer, satisfies

$$
U_{\ell}(N)=o(1) \quad \text { as } N \rightarrow \infty \text {. }
$$

Setting

$$
\sigma_{N}(k):=\#\left\{p \leq N: s_{q}(p)=k\right\}
$$

we have

$$
\begin{aligned}
U_{\ell}(N) & =\sum_{k \equiv \ell} e(m f(k)) \sigma_{N}(k) \\
& =\sum_{t \geq 0} e(m f(t(q-1)+\ell)) \sigma_{N}(t(q-1)+\ell) .
\end{aligned}
$$

Observe that the leading coefficient of the above polynomial $f(t(q-1)+\ell)$ is $\alpha(q-1)^{k}$, which is a non Liouville number as well (as we mentioned in the proof of Theorem 1), and also that the functions

$$
w_{N}(t):=\frac{1}{\Pi_{\ell}(N)} \sigma_{N}(t(q-1)+\ell)
$$

may be considered as weights (since $\sum_{k=1}^{\infty} w_{N}(t)=1$ ). Thus, applying Lemma 2, we obtain (17), thereby completing the proof of Theorem 2. 


\section{Proof of Theorem 3}

We shall skip the proof of estimate (7), since it can be obtained along the same lines as that of the main theorem in De Koninck, Kátai and Phong [2].

In order to obtain $(8)$, we separate the set $\wp$ into $\varphi(q-1)$ distinct sets $\wp_{\ell}$, with corresponding counting function $\Pi_{N}(\ell)$ defined in (15).

Observe that

$$
g\left(\alpha Q^{t(q-1)+\ell}\right) \sigma_{N}(t(q-1)+\ell)=g\left(\left(\alpha Q^{\ell}\right) \cdot Q^{t(q-1)}\right) \sigma_{N}(t(q-1)+\ell)
$$

Now, since $\alpha$ is a strongly $Q$-normal number, then so is $\alpha Q^{\ell}$, a number which is strongly $Q^{q-1}$-normal.

We then have

$$
\begin{aligned}
& \sum_{p \leq N} g\left(\alpha Q^{s_{q}(p)}\right)=\sum_{k \geq 1} \sum_{\substack{p \leq N \\
s q(p)=k}} g\left(\alpha Q^{k}\right) \\
& =\sum_{\substack{\ell=1 \\
(\ell, q-1)=1}}^{q-1} \sum_{\substack{p \leq N \\
p \in \mathcal{P}_{\ell}}} g\left(\alpha Q^{t(q-1)+\ell}\right) \sigma_{N}(t(q-1)+\ell) \\
& =\sum_{\substack{\ell=1 \\
(\ell, q-1)=1}}^{q-1} \sum_{\substack{p \leq N \\
p \in \mathcal{Q}_{\ell}}} g\left(\left(\alpha Q^{\ell}\right) \cdot Q^{t(q-1)}\right) \sigma_{N}(t(q-1)+\ell) .
\end{aligned}
$$

Since we then have

$$
\lim _{N \rightarrow \infty} \frac{1}{\Pi_{\ell}(N)} \sum_{\substack{p \leq N \\ p \in \mathfrak{Q}_{\ell}}} g\left(\alpha Q^{s_{q}(p)}\right)=0 \quad \text { for each } \ell \text { with }(\ell, q-1)=1,
$$

summing up over all $\ell$ 's such that $(\ell, q-1)=1$, estimate (8) follows immediately.

\section{References}

[1] J.M. De Koninck and I. Kátai, On the distribution modulo 1 of the values of $F(n)+\alpha \sigma(n)$, Publicationes Mathematicae Debrecen 66 (2005), 121-128.

[2] J.M. De Koninck, I. Kátai and B.M. Phong, On strong normality, preprint.

[3] J.M.De Koninck and F. Luca, Analytic Number Theory: Exploring the Anatomy of Integers, Graduate Studies in Mathematics, Vol. 134, American Mathematical Society, Providence, Rhode Island, 2012.

[4] H. Delange, Sur la fonction sommatoire de la fonction "somme des chiffres", Enseign. Math. (2) 21.1 (1975), 31-47.

[5] M. Drmota and J. Gajdosik, The distribution of the sum-of-digits function, J. Théor. Nombres Bordeaux 10 (1998), No. 1, 17-32. 
[6] M. Drmota, P.J. Grabner and P. Liardet, Block additive functions on the Gaussian integers, Acta Arith. 135 (2008), No. 4, 299-332.

[7] M. Drmota and J. Rivat, The sum of digits function of squares, J. London Math. Soc. 72 (2005), 273-292.

[8] M. Drmota, C. Mauduit and J. Rivat, Primes with an average sum of digits, Compos. Math. 145 (2009), No. 2, 271-292.

[9] M. Drmota, C. Mauduit and J. Rivat, The sum of digits function of polynomial sequences, J. Lond. Math. Soc. (2) 84 (2011), No. 1, 81-102.

[10] A. O. Gelfond, Sur des nombres qui ont des propriétés additives et multiplicatives données, Acta Arith. 13 (1968), 259-265.

[11] G. Halász, On the distribution of additive and the mean values of multiplicative arithmetic functions, Studia Sci. Math. Hungar. 6 (1971), 211-233.

[12] I. Kátai, A remark on a paper of K. Ramachandra in Number theory (Ootacamund, 1984), 147-152, Lecture Notes in Math., 1122, Springer, Berlin, 1985.

[13] C. Mauduit and J. Rivat, Sur un problème de Gelfond: la somme des chiffres des nombres premiers, Ann. of Math. (2) 171 (2010), no. 3, 1591-1646.

[14] C. Mauduit and J. Rivat, La somme des chiffres des carrés, Acta Math. 203 (2009), No. 1, 107-148.

[15] J. F. Morgenbesser, The sum of digits of squares in $\mathbb{Z}[i]$, J. Number Theory 130 (2010), 1433-1469.

[16] R. C. Vaughan, The Hardy-Littlewood method, Bull. Amer. Math. Soc. (N.S.) 7 (1982), no. 2, 433-437. 